University of Nebraska - Lincoln

DigitalCommons@University of Nebraska - Lincoln

Norman R. Simon Papers

Research Papers in Physics and Astronomy

$9-1-1984$

\title{
Phase Lags and Pulsation Modes of Classical Cepheids
}

Norman R. Simon

University of Nebraska - Lincoln, nsimon@unl.edu

Follow this and additional works at: https://digitalcommons.unl.edu/physicssimon

Simon, Norman R., "Phase Lags and Pulsation Modes of Classical Cepheids" (1984). Norman R. Simon Papers. 19.

https://digitalcommons.unl.edu/physicssimon/19

This Article is brought to you for free and open access by the Research Papers in Physics and Astronomy at DigitalCommons@University of Nebraska - Lincoln. It has been accepted for inclusion in Norman R. Simon Papers by an authorized administrator of DigitalCommons@University of Nebraska - Lincoln. 


\title{
PHASE LAGS AND PULSATION MODES OF CLASSICAL CEPHEIDS
}

\author{
NoRman R. Simon \\ Department of Physics and Astronomy, University of Nebraska-Lincoln \\ Received 1983 October 31; accepted 1984 March 20
}

\begin{abstract}
The first-order phase lag between light and velocity is calculated from Fourier decompositions of the variations of 12 classical Cepheids. The phase lags have a narrow range, with maximum expansion velocity always retarded with respect to maximum light. The average value of the first-order phase lag is found to be approximately -0.3 , close to the theoretical value obtained from the models of Simon and Davis. When the phase lag is plotted against period, two of the stars stand out. These stars are SU Cas and AZ Cen, both suspected overtone pulsators. We further investigate $\mathrm{AZ} \mathrm{Cen} \mathrm{by} \mathrm{combining} \mathrm{the} \mathrm{light} \mathrm{curves} \mathrm{of} \mathrm{three} \mathrm{observers} \mathrm{and} \mathrm{plot-}$ ting the star on the $\phi_{21}$-period diagram of Simon and Lee. The tentative result is seen to strengthen both the identification of $\mathrm{AZ} \mathrm{Cen} \mathrm{as} \mathrm{an} \mathrm{overtone} \mathrm{pulsator} \mathrm{and} \mathrm{the} \mathrm{possible} \mathrm{role} \mathrm{of} \mathrm{the} \mathrm{first-order} \mathrm{phase} \mathrm{lag} \mathrm{as} \mathrm{a} \mathrm{dis-}$ criminator of mode. We close with a discussion of our results and of the uncertainties which remain.
\end{abstract}

Subject headings: stars: Cepheids - stars: pulsation

\section{INTRODUCTION}

It is well known that the light and velocity curves of classical Cepheids are approximately "mirror images" with maximum light occurring very close to the phase of maximum velocity of expansion. However, the literature contains few precise measurements of the degree to which the light actually leads or lags the velocity in individual stars. According to Ledoux and Walraven (1958), the velocity curve seems to be retarded with respect to the light by an amount which is generally less than a tenth of the period and which seems to be larger for the longer period stars.

Carson and Stothers (1984) have compiled or calculated phase lags for a selection of long-period Cepheids $(P>14$ days). They also find the velocity lagging the light in all caseson the average by somewhat more than $10 \%$ of the period, but with considerable scatter. These authors quote a typical error in the phase lag of $2 \%$ to $5 \%$ of the period. A number of stars in the Carson-Stothers sample were taken from Evans (1976) who reports an error of $2 \%$ of the period. However, in most of the shorter period Cepheids observed by Evans, the velocity was determined to lead (rather than lag) the light.

On the theoretical side, Stobie $(1969 a, b)$ constructed a large number of hydrodynamic models and examined their light and velocity curves. He found that, in most cases, the velocity curves were advanced (rather than retarded) with respect to the light. However, Stobie's definition of the phase lag was static luminosity minus zero velocity on the ascending branch of the light curve, quite different from the definition employed by Ledoux and Walraven (1958) and Carson and Stothers (1984). Stobie $(1969 b)$ also investigated the effects of composition changes on the phase lag, finding that the advance of velocity over light was increased by increases in both the helium and heavy element abundances. He suggested that element abundances might be determined from observed phase lags but did not attempt to do this in his own study.

More recently, Simon and Davis (1983) computed a series of Cepheid light and velocity curves using both hydrodynamic and linear nonadiabatic (LNA) pulsation codes. Three versions of the phase lag (defined as maximum light minus maximum expansion velocity, in radians) were calculated from these models: (1) the full nonlinear phase lag, $\Delta \phi$, read off from the hydrodynamic light and velocity curves; (2) the phase lag, $(\Delta \phi)_{\text {LNA }}$, coming from the LNA models; and (3) the first-order phase lag, $(\Delta \phi)_{1}$, computed from the phase difference between the lowest order terms in the Fourier decomposition of the light and velocity curves respectively. While both $\Delta \phi$ and $(\Delta \phi)_{\text {LNA }}$ showed large scatter from model to model about the "canonical" value $\Delta \phi=0$, the quantity $(\Delta \phi)_{1}$ varied little among the models, having a typical value $(\Delta \phi)_{1} \approx-0.3$. This corresponds to the velocity lagging the light by about $5 \%$ of the period.

\section{OBSERVED PHASE LAGS}

Table 1 lists 12 classical Cepheids which were the most promising candidates we could find in the literature for the calculation of phase lags. Each of these objects was either observed simultaneously in light and velocity or was shown to have a period which did not vary during the time lapse between the light and velocity measurements. Successive

TABLE 1

The ObSERVATIONAL SAMPLE

\begin{tabular}{|c|c|c|c|c|}
\hline Star Name & Source & Period & $\begin{array}{l}\text { Amp. } \\
\text { (mag) }\end{array}$ & $(\Delta \phi)_{1}$ \\
\hline SU Cas. & 1 & 1.949325 & 0.42 & -0.48 \\
\hline $\mathrm{AZ}$ Cen $\ldots .$. & $2,3,4$ & 3.212055 & 0.37 & -0.56 \\
\hline $\mathrm{R} \operatorname{Tr} \mathrm{A} \ldots \ldots$ & 2,3 & 3.389287 & 0.54 & -0.29 \\
\hline SS Sct $\ldots \ldots \ldots$ & 2,3 & 3.671253 & 0.51 & -0.14 \\
\hline $\mathrm{AD} \mathrm{Gem} \ldots$ & 5 & 3.787980 & 0.68 & -0.22 \\
\hline $\mathrm{XY}$ Cas ......... & 6 & 4.501697 & 0.59 & -0.18 \\
\hline V482 Sco .... & 2,3 & 4.52786 & 0.64 & -0.22 \\
\hline V Cen ........ & 2,3 & 5.49392 & 0.76 & -0.15 \\
\hline S TrA ......... & 2,3 & 6.32344 & 0.71 & -0.19 \\
\hline BB Sgr $\ldots \ldots$ & 2,3 & 6.63699 & 0.60 & -0.39 \\
\hline U Sgr $\ldots \ldots \ldots$ & 2,3 & 6.744925 & 0.73 & -0.27 \\
\hline V496 Aql .... & 2,3 & 6.80703 & 0.38 & -0.43 \\
\hline
\end{tabular}

SOURCES-11) Abt 1959, Gieren 1976, Beavers 1979 (velocity); Mitchell, et al. 1964, Niva and Schmidt 1979 (light). (2) Gieren $1981 a$ (velocity). (3) Gieren $1981 b$ (light). (4) Stobie and Balona 1979 (velocity); Pel 1976, Stobie and Balona 1979 (light). (5) Imbert 1983. (6) Imbert 1981. 


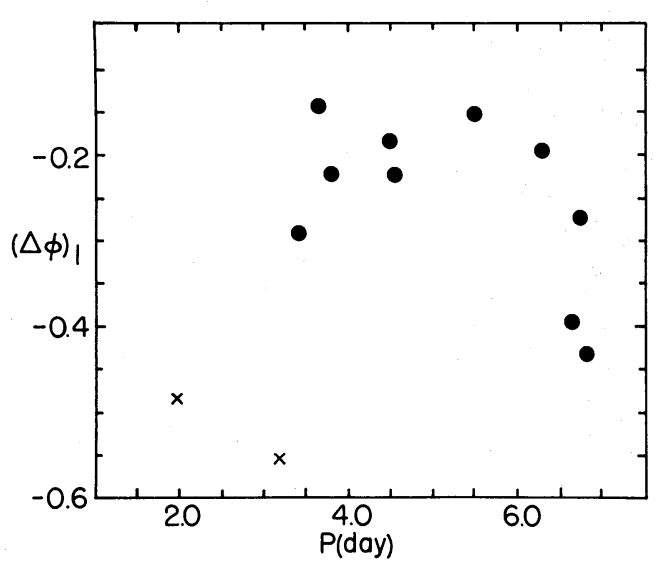

FIG. 1.-The first-order phase $\operatorname{lag}(\Delta \phi)_{1}$ vs. period. Crosses indicate the two suspected overtone pulsators, SU Cas and AZ Cen.

columns in Table 1 give the star name, references for the data, the period, the light amplitude in magnitudes, and the firstorder phase lag, $(\Delta \phi)_{1}$, calculated from Fourier decompositions of the observed light and velocity curves according to the expression

$$
(\Delta \phi)_{1}=\phi_{1}(\text { velocity })-\phi_{1}(\text { magnitude })+\pi / 2,
$$

where $\phi_{1}$ (magnitude) and $\phi_{1}$ (velocity) are respectively defined by equations (1) and (2) of Simon and Davis (1983).

Figure 1 shows a plot of $(\Delta \phi)_{1}$ versus period for the 12 stars in our sample. The values of $(\Delta \phi)_{1}$ are all negative and have the range $-0.56 \leq(\Delta \phi)_{1} \leq-0.14$, with an average value $(\Delta \phi)_{1}=$ -0.29 . Thus the observational first-order phase lags agree reasonably well with those coming from the models of Simon and Davis (1983). The latter had the range $-0.35 \leq(\Delta \phi)_{1} \leq+0.03$ with an average value $(\Delta \phi)_{1}=-0.26$.

\section{PULSATION MODE}

At the short-period end of Figure 1, two points lie noticeably below the others - these are SU Cas and AZ Cen. SU Cas is strongly suspected of being an overtone pulsator (Gieren 1982a), not the least because of its anomalous position in the $\phi_{21}$-period diagram of Simon and Lee (1981). AZ Cen is also a suspected overtone (Gieren 1982b), but the evidence is weaker.

The literature contains a number of observations of AZ Cen, including those by Pel (1976), Gieren (1981 $a, b)$, and Stobie and Balona (1979). Gieren adopts the period given by Pel, while Stobie and Balona quote a somewhat different period. When we attempted to merge the various data sets using the former period, serious discrepancies arose. However, when the latter value was employed, the data combined quite well. Therefore, we have adopted the period given by Stobie and Balona and have entered that period in Table 1. When Fourier decompositions were performed on the combined light curve of AZ Cen, we were able to determine the quantity $\phi_{21}$ (Simon and Lee 1981):

$$
\phi_{21}(\mathrm{AZ} \text { Cen }) \approx 4.5
$$

This value, if correct, puts AZ Cen well above other stars of its period on the $\phi_{21}$-period diagram and thus strengthens both the identification of $\mathrm{AZ}$ Cen as an overtone pulsator and the idea that the first-order phase lag, $(\Delta \phi)_{1}$, may be used as an indicator of pulsation mode.

However, there are a number of cautionary notes which should be inserted here. In the first place, the amplitude of the leading harmonic term (frequency $=2 \omega$ ) in the Fourier fit for AZ Cen is very small, approximately $0.01 \mathrm{mag}$, a value slightly less than the standard deviation of the fit itself. Thus the value of $\phi_{21}$ quoted above has, in effect, been retrieved from the noise. Second, the suspicion has been voiced by Pel (1976) that the light curve of AZ Cen may be variable in a way reminiscent of the double-mode Cepheids. To test this suggestion, we have attempted to fit the combined light curve with a two-mode, Fourier-interaction series (e.g., Simon 1979) assuming alternately that the dominant mode is the fundamental and that it is the first overtone. While we could not find any evidence for a second mode in either case, we cannot rule out the presence of such a mode at an amplitude of a few thousandths of a magnitude.

Another bit of evidence supporting the possible role of $(\Delta \phi)_{1}$ as a mode discriminator comes from Fourier decompositions of the light and velocity curves of the double-mode dwarf Cepheid, AI Velorum (Simon 1979). The phase lag, $(\Delta \phi)_{1}$, was calculated for each mode, with the result

$$
\text { AI Vel: }(\Delta \phi)_{1}^{\text {fund }}=-0.10 ;(\Delta \phi)_{1}^{\text {over }}=-0.44
$$

We note that the relationship between the overtone and fundamental phase lags for AI Vel is reminiscent of that found above for SU Cas and AZ Cen versus the rest of the short-period Cepheid sample. This again suggests that SU Cas and AZ Cen may indeed be overtone pulsators.

\section{DISCUSSION}

We begin by pointing out that the velocity observations for nine of the 12 stars in our sample were examined in connection with an earlier investigation by Simon and Teays (1983). Three of these nine stars were ultimately treated by those authors, who considered the data extensive enough to determine the second-order quantities $\phi_{21}$ and $R_{21}$; the other six data sets were rejected. In the present study, however, we are able to include the six rejected stars (AZ Cen, SS Sct, V Cen, BB Sgr, U Sgr, and V496 Aql) because calculation of the phase lag $(\Delta \phi)_{1}$ requires only first-order quantities in the Fourier decompositions.

The full nonlinear phase lag, $\Delta \phi$, can be obtained from the observational data by reconstructing the light and velocity curves from the Fourier coefficients and noting the phases of maximum light and maximum velocity of expansion. Figure 2 shows a plot of $\Delta \phi$ versus $(\Delta \phi)_{1}$. While for the purpose of completeness (and to avoid manipulating the data) we have included all 12 stars in this diagram, the six objects named above have been plotted as crosses. We do not consider the values of $\Delta \phi$ for these stars to be completely reliable. However, we may note two things from our small sample: (1) the correlation between $\Delta \phi$ and $(\Delta \phi)_{1}$ is considerably better than that obtained for the theoretical models treated by Simon and Davis (1983); and (2) with one exception the values of $\Delta \phi$ are all zero or less. Thus we see that the phase lags, $\Delta \phi$, obtained in the present work disagree with those given for the short-period sample of Evans (1976), even within the quoted errors. Because the stars we treated were specially selected as described above, and because our method of determining phase lags is simple and straightforward, it is our opinion that the values determined here are essentially correct. Since none of the stars studied by Evans are included in our own sample, it is possible that her phase lags are also correct, in which case there seem to exist different classes (with respect to phase lag) among short- 
period Cepheids. While this does not seem likely, the possibility needs to be checked with further observations.

Among recent theoretical calculations, both the Los Alamos models (Simon and Davis 1983) and the available Carson opacity models (Carson and Stothers 1984; Vemury and Stothers 1978) tend to have periods longer than those of the present observational sample. In fact, among 26 theoretical models (14 from Simon and Davis 1983, eight from Carson and Stothers 1984, and four from Vemury and Stothers 1978 as treated by Simon and Davis 1983), only two have periods in the range of the present sample- the 5.30 day model of Simon and Davis and the 6.85 day model of Vemury and Stothers. Interestingly enough, in these two cases the phase lags $(\Delta \phi)_{1}$ and $\Delta \phi$ agree quite well with each other and also with the observational values obtained in the present investigation.

Figure 3 shows a plot of $(\Delta \phi)_{1}$ versus the light amplitude for the 12 stars of our sample. While it is possible to discern what might be a trend of increasing $(\Delta \phi)_{1}$ with amplitude, we do not feel our sample is large enough to make a reliable statement in this regard. (We note that Carson and Stothers 1984 found evidence for a similar trend in $\Delta \phi$ among some of their theoretical models.) This point is important, however, since if this trend exists, then SU Cas and AZ Cen could have small (i.e., more negative) values of $(\Delta \phi)_{1}$ not produced by any (presumed) overtone nature but merely by their low amplitudes. In that case, short-period, low-amplitude, fundamental mode pulsators like VZ CMa and BY Cas (Simon and Lee 1981) also ought to show small values of $(\Delta \phi)_{1}$. This again can be checked by further observations.

\section{FUTURE WORK}

Two potentially important results emerge from the Fourier decompositions performed in the present study. In the first

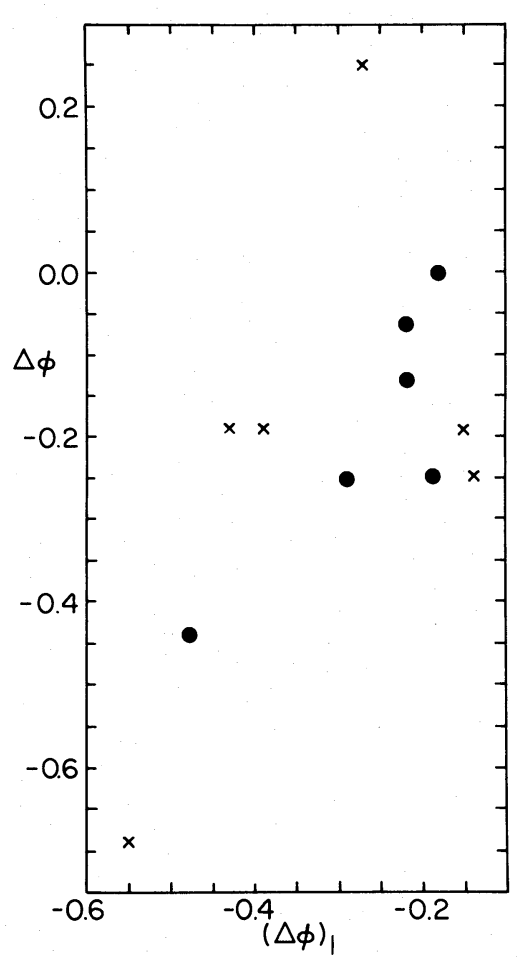

Fig. 2. - The full phase lag $\Delta \phi$ vs. $(\Delta \phi)_{1}$. Points indicated by crosses are not considered completely reliable (see the text).

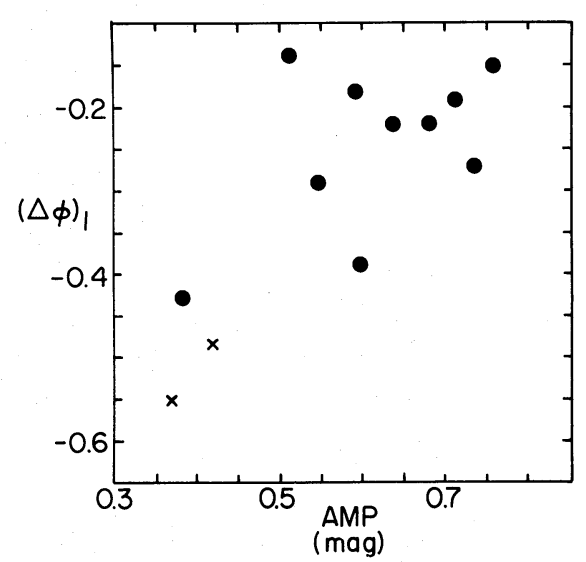

Fig. 3. - The first-order phase lag $(\Delta \phi)_{1}$ vs. the light amplitude. Crosses denote SU Cas and AZ Cen.

place, the first-order phase lag has been shown to be negative in all cases with an average value $(\Delta \phi)_{1} \approx-0.3$. This value was reproduced well by the hydrodynamic integrations by Simon and Davis (1983) but not by LNA models with the same parameters. Thus, contrary to the conclusion of Simon (1979), it appears that nonlinear effects must play an important role in $(\Delta \phi)_{1}$.

In this context, the first-order phase lag could prove helpful in evaluating hydrodynamic models. At the outset, it would be very useful to obtain both suitable observations of longer period stars and hydrodynamic calculations for shorter period objects in order to extend the overlap between observed and theoretical phase lag data. In addition, it would be interesting to see what effect the inclusion of convection in hydrodynamic models will have on $(\Delta \phi)_{1}$. Since it is known that phase lags can be severely altered by including convection, at least in LNA models (Baker and Kippenhahn 1965), the quantity $(\Delta \phi)_{1}$ might play a role as guide to the proper formulation of convection in nonlinear pulsation codes (e.g., Stellingwerf 1982). Finally, one should examine the effect of element abundances (and/or opacity) on $(\Delta \phi)_{1}$ to explore its possible use as a probe of chemical composition as suggested by Stobie $(1969 b)$.

The second possibly important finding of the present investigation concerns the employment of $(\Delta \phi)_{1}$ as a mode discriminator. We have shown that the suspected overtone pulsators SU Cas and AZ Cen are separated from the other stars on a plot of $(\Delta \phi)_{1}$ versus period. Furthermore, by combining three data sets we have provided further tentative evidence for the overtone nature of AZ Cen.

At the moment, however, we cannot rule out the possibility that $(\Delta \phi)_{1}$ is governed by amplitude rather than pulsation mode. Should this turn out to be the case, the relationship between phase lag and amplitude could provide yet another test for theoretical models. However, because our sample is small and the data often sparse, further observations will be necessary to firmly establish an important role for $(\Delta \phi)_{1}$ in the study of pulsating stars.

We are pleased to acknowledge support from the National Science Foundation under grants AST 81-05064 and AST 8316875 . We are also grateful to an anonymous referee for pointing out an error in an earlier version of this work. 
REFERENCES

Mitchell, R. I., Iriarte, B., Steinmetz, D., and Johnson, H. L. 1964, Bol. Inst. Tonantzintla, 3, 153 .

Baker, N. H and Kippenhahn, R. 1965, Ap. J., 142, 868

I Beavers, W. I. 1979, private communication.

Io' Carson, T. R., and Stothers, R. B. 1984, Ap. J., 276, 593.

Evans, N. R. 1976, Ap. J. Suppl., 32, 399.

- Gieren, W. 1976, Astr. Ap., 47,211.

1981a, Ap. J. Suppl., 46, 287.

1981 b, Ap. J.Suppl, 47, 315.

1982a, Pub. A.S.P., 94, 960

$1982 b$, Ap. J., 260, 208.

Imbert, M. 1981, Astr. Ap. Suppl., 44, 319.

1983, Astr. Ap. Suppl., 53, 85

Ledoux, P., and Walraven, Th. 1958, in Handbuch der Physik, ed. S. Flugge (Berlin: Springer), 51, 353 .

Niva, G. D and Schmidt, E. G. 1979 , Ap. J. 234, 245.

Pel, J. W. 1976, Astr. Ap. Suppl., 24, 413.

Simon, N. R. 1979, Astr. Ap., 74, 30

Simon, N. R., and Davis, C. G. 1983, Ap. J., 266, 787

Simon, N. R., and Lee, A. S. 1981, Ap. J., 248, 291.

Simon, N. R. and Teays, T. J. 1983, Ap. J., 265, 996.

Stobie, R. S. $1969 a$, M.N.R.A.S., 144, 485

.1969b, M.N.R.A.S., 144, 511.

Stobie, R. S., and Balona, L. A. 1979, M.N.R.A.S., 189, 641

Stellingwerf, R. F. 1982, Ap. J., 262, 339.

Vemury, S. K., and Stothers, R. 1978, Ap. J., 225, 939.

NoRman R. Simon: Department of Physics and Astronomy, University of Nebraska-Lincoln, Lincoln, NE 68588 